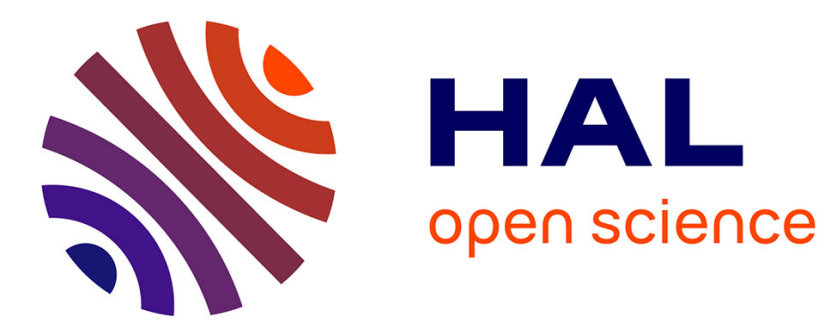

\title{
Surfaces et interfaces prototypes du silicium et du germanium
}

\author{
G. Le Lay
}

\section{To cite this version:}

G. Le Lay. Surfaces et interfaces prototypes du silicium et du germanium. Journal de Physique IV Proceedings, 1997, 07 (C6), pp.C6-115-C6-125. 10.1051/jp4:1997610 . jpa-00255709

\section{HAL Id: jpa-00255709 https://hal.science/jpa-00255709}

Submitted on 1 Jan 1997

HAL is a multi-disciplinary open access archive for the deposit and dissemination of scientific research documents, whether they are published or not. The documents may come from teaching and research institutions in France or abroad, or from public or private research centers.
L'archive ouverte pluridisciplinaire HAL, est destinée au dépôt et à la diffusion de documents scientifiques de niveau recherche, publiés ou non, émanant des établissements d'enseignement et de recherche français ou étrangers, des laboratoires publics ou privés. 


\title{
Surfaces et interfaces prototypes du silicium et du germanium
}

\author{
G. Le Lay \\ CRMC2-CNRS, Campus de Luminy, Case 913, 13288 Marseille cedex 09, France, et, UFR Sciences de la \\ Matière, Université de Provence, Marseille, France
}

\begin{abstract}
Résumé. Nous đécrivons dans cet article quelques phénomènes particuliers, découverts récemment aux surfaces (111) et (100) du silicium et du germanium recouvertes de films ultra-minces de métaux non-réactifs : l'étain, le plomb, le bismuth ainsi qu'à leurs interfaces "prototypes" pour des couches métalliques épaisses.
\end{abstract}

\begin{abstract}
We describe in this paper a few peculiar phenomena recently discovered at the (111) and (100) surfaces of silicon and germanium covered with ultra-thin non-reactive metal overlayers : tin, lead and bismuth as well as at their "prototypical" interfaces for thick metal films.
\end{abstract}

\section{INTRODUCTION}

La plupart des métaux réagissent fortement avec le silicium ou le germanium. Seules quelques exceptions, telles que le plomb, l'étain ou le bismuth dérogent à la règle et conduisent à des interfaces abruptes. Les systèmes "prototypes" ainsi formés donnent lieu à d'étonnants phénomènes, découverts récemment. Certains de ces phénomènes, directement reliés à la basse dimensionnalité, seront décrits dans cet article. Ils concernent aussi bien les arrangements atomiques aux surfaces et aux interfaces que les structures électroniques qui leurs sont associées. Leur découverte tient souvent à l'utilisation en synergie de méthodes avancées d'observation et d'analyse mettant en jeu d'un côté les sondes locales telles que la microscopie et la spectroscopie à effet tunnel, d'un autre côté les moyens de caractérisation qu'offrent en particulier les synchrotrons de troisième génération.

Nous montrerons ainsi que la mise en évidence de fluctuations métal/semiconducteur à température ambiante au sein des adatomes d'étain constituant les reconstructions $\mathrm{Si}, \mathrm{Ge}(111) \sqrt{3} \times \sqrt{3}-\mathrm{R}\left(30^{\circ}\right)-\mathrm{Sn}$ alpha (en bref $\mathrm{R} 3 \alpha$ ) [1] anticipe déjà la formation d'une onde de densité de charge de surface découverte peu après pour le système voisin plomb-germanium (111) à basse température [2]. Toujours dans le domaine de la sous-monocouche, cette fois sur la face (100) du silicium, le plomb forme initialement de longues rangées unidimentionnelles de dimères [3].

A plus fort recouvrement une structure $2 \times 1$ se développe [4] comme aveč le bismuth [5]. Dans ce dernier cas la structure est composée de dimères de bismuth adsorbés sur un substrat, tel qu'idéalement tronqué en volume.

La structure initiale de surface peut soit perdurer, soit disparaître sous des films épais de métal, mais la structure interfaciale influe alors directement sur la hauteur de barrière de Schottky [6]. Dans ce contexte la présence intentionnelle d'une couche intermédiaire d'hydrogène à l'interface $\mathrm{Pb} / \mathrm{Si}(111) \mathrm{1x} 1-\mathrm{H}$ joue un rôle considérable $[7,8]$.

Ces exemples illustrent de façon non exhaustive la variété des phénomènes inattendus observés depuis peu aux surfaces et interfaces de systèmes métal/semiconducteur prototypes. Une présentation plus complète de ces phénomènes constitue un chapitre de l'ouvrage collectif : "Growth and Properties of Ultrathin Epitaxial Layers" [9].

\section{LES PHASES R3 ALPHA}

Au taux de recouvrement d'un tiers de monocouche $(\theta=1 / 3 \mathrm{MC})$ le plomb et l'étain forment une structure $\mathrm{R} 3$ commune notée $\alpha$ pour la distinguer des phases mosaïques $(\theta=1 / 6 \mathrm{MC})$ notées $\gamma$ et des phases 
obtenues à saturation, notées $\beta$. La Figure 1 donne la structure de deux de ces phases pour le système $\mathrm{Pb} / \mathrm{Ge}(111)$.

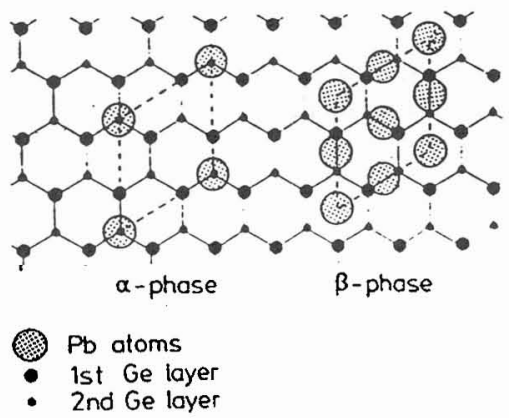

Figure 1 : Modèles atomiques des structures $\mathrm{R} 3 \alpha$ et $\beta$ du système $\mathrm{Pb} / \mathrm{Ge}(111)$

La structure $\mathrm{R} 3 \alpha$, déterminée par diffraction des rayons $\mathrm{X}$ sous incidence rasante en rayonnement synchrotron [10] peut être décrite à partir d'un réseau (111) tronqué en volume, décoré par 1/3 de monocouche d'adatomes de $\mathrm{Pb}$ ou $\mathrm{Sn}$ en sites $\mathrm{T}_{4}$.

\subsection{Fluctuations métal-semiconducteur à température ambiante}

Comparant les largeurs des niveaux de coeur $\mathrm{Pb} 5 \mathrm{~d}$ pour les trois structures $\mathrm{R} 3 \mathrm{du}$ système $\mathrm{Pb} / \mathrm{Ge}(111)$, Si(111), Carlisle et al. [11,12] relevaient un comportement surprenant que la figure 2 illustre.
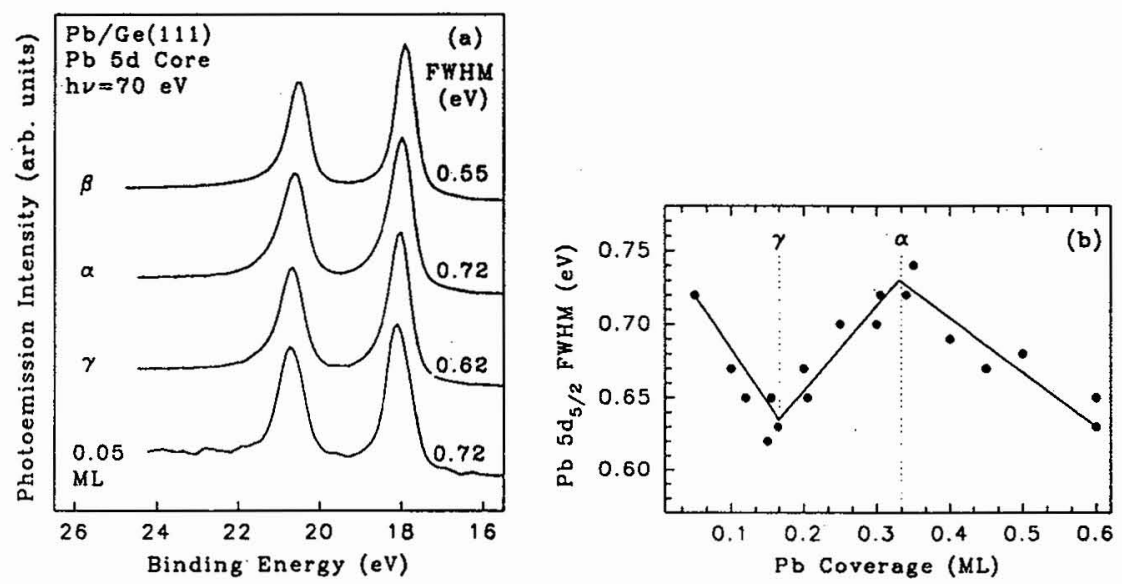

Figure 2 : a) Comparaison des niveaux de coeur $\mathrm{Pb} 5 \mathrm{~d}$ mesurés pour les phases $\mathrm{R} 3 \gamma, \alpha$ et $\beta$ et un très faible recouvrement de $0,05 \mathrm{MC}$.

b) Variation de la largeur à mi-hauteur du pic $5 \mathrm{~d} 5 / 2$ en fonction du taux de recouvrement (d'après [11]).

Il pourrait être tentant d'attribuer les variations de largeur mesurées à la présence de plusieurs composantes non résolues du fait de l'existence éventuelle de sites inéquivalents ou d'un degré d'ordre variable de ces surfaces. Cependant on note paradoxalement que le maximum de largeur correspond juste à la phase $\alpha$ qui donne les meilleurs clichés de diffraction, les états de surface les plus fins dans les spectres de bande de valence et surtout qui ne comprend qu'un site d'adsorption unique, le site $\mathrm{T}_{4}$. 
Nous avons pour notre part mesuré les niveaux de coeurs des systèmes $\mathrm{Sn} / \mathrm{Si}(111), \mathrm{Ge}(111)$ pour les phases mosaïques et $\alpha$ sur la ligne à haute résolution énergétique dotée d'un monochromateur de type SX 700 de MAX-lab à Lund (Suède) [1]. Alors que les surfaces R3 observées en microscopie à effet tunnel [13] (fig. 3a) apparaissent quasi parfaites si ce n'est pour la présence de quelques adatomes substitutionnels de germanium répartis au hasard, ce qui laisserait présager une seule composante pour les niveaux de coeur $\mathrm{Sn} 4 \mathrm{~d}$, on observe au contraire qu'au moins deux contributions interviennent (cf. Fig. $3 \mathrm{~d})$. Ce fait est à rapprocher de l'élargissement des niveaux $\mathrm{Pb} 5 \mathrm{~d}$ noté ci-dessus. Comme les intensités relatives des deux composantes D1 et D2 utilisées pour la synthèse numérique du spectre de la figure $3 \mathrm{~b}$ restent inchangées pour des angles d'émission hors de la normale, il est clair que ces deux composantes proviennent de la couche la plus externe d'adatomes. Ces intensités sont trop proches pour être reliées à des environnements différents (c'est à dire proches voisins ou pas d'un adatome de germanium substitutionnel). Par ailleurs, on ne note aucune différence significative entre adatomes de même nature dans les images de microscopie tunnel. Il paraît donc improbable que l'écart énergétique relativement important entre ces deux composantes soit également à relier à des sites différents.
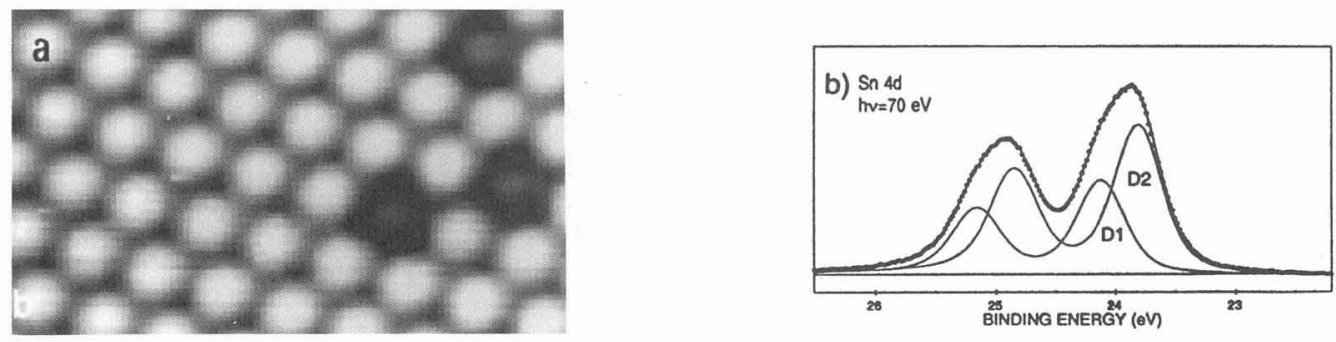

Figure 3 : a) Image de microscopie à effet tunnel de la structure Ge(111) R3 $\alpha-S n(1 \mathrm{nA}$, polarisation + 1V de l'échantillon vis- à-vis de la pointe) ; b) Spectre de photoemission du niveau de coeur $\mathrm{Sn} 4$ d correspondant, mesuré à $\sim 100 \mathrm{~K}$ en émission normale (d'après [13]).

Les structures $\mathrm{R} 3 \alpha$ sont métalliques, comme l'on peut s'y attendre d'après le décompte du nombre d'électrons, puisque chaque atome du groupe IV a une liaison pendante insaturée. Comme on peut le voir sur la figure 4a, le niveau de Fermi de la surface $\mathrm{Si}(111) \mathrm{R} 3 \alpha$-Sn traverse du côté des hautes énergies cinétiques l'état de surface $\mathrm{S} 1$ associé à la bande à moitié pleine des liaisons pendantes des adatomes. La phase mosaïque est au contraire semiconductrice puisque la structure S1 est située dans ce cas nettement au-dessous du niveau de Fermi.

Ce passage d'un caractère métallique à semiconducteur reflète le transfert de charge, déduit des images tunnel obtenues à des tensions différentes [14], de la liaison pendante des adatomes de silicium ( $50 \%$ des adatomes dans la phase mosaïque) vers celle des adatomes d'étain. L'effet le plus remarquable est la spectaculaire diminution de largeur des niveaux de coeurs Sn 4d [15] (Fig. 4b).

Le spectre de la phase mosaïque peut être ajusté avec une seule composante coïncidant avec la contribution à plus faible énergie de liaison, D2, (voir figure 3b) du spectre de niveau de coeur $\mathrm{Sn} 4 \mathrm{~d}$ de la phase R3 $\alpha$.

Pour élucider l'origine mystérieuse des deux composantes, D1 et D2, nous avons utilisé le potassium à la fois comme entité sonde et perturbatrice de la structure électronique.

L'adsorption à température ambiante d'environ $1 / 3 \mathrm{MC}$ de potassium, transforme le spectre Sn $4 d$ à deux composantes de la phase métallique $\mathrm{R} 3 \alpha\left(\mathrm{T}_{4}\right)$ en un spectre analogue à celui de la phase mosaïque (figure 5). Corrélativement la largeur de la Gaussienne des raies du niveau de coeur Si $2 p$ décroit très fortement et la surface devient semiconductrice. 
a)

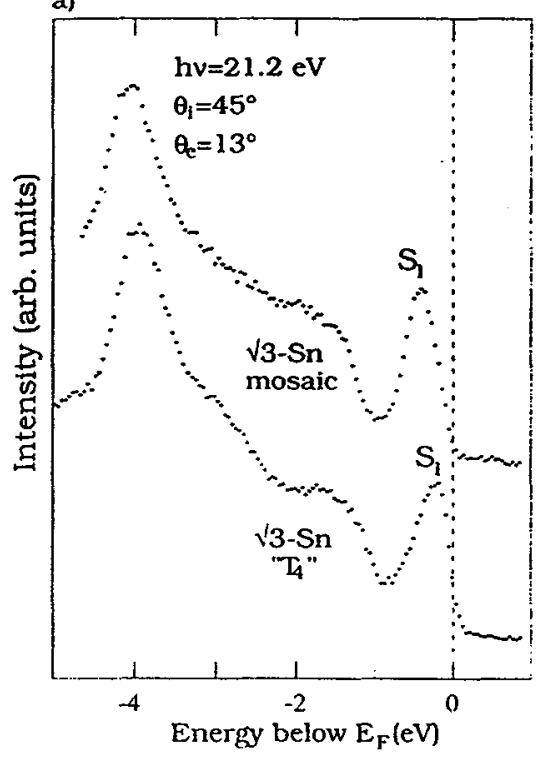

b)

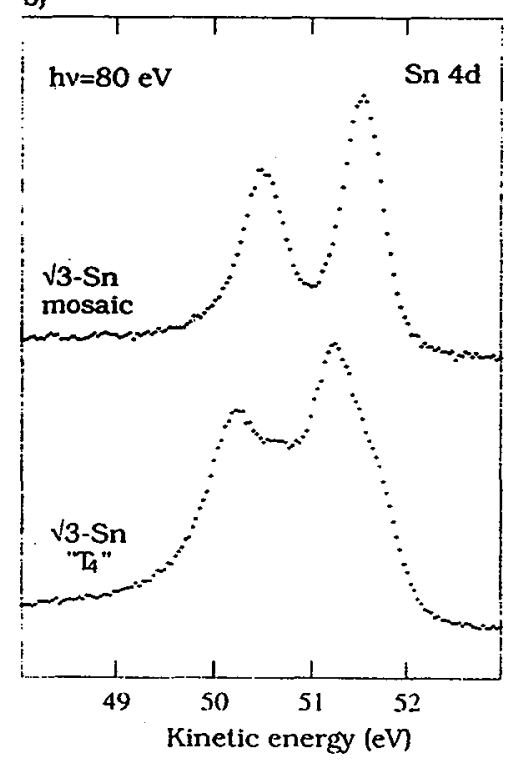

Figure 4 : a) Spectres de bande de valence de la phase $\mathrm{R} 3 \alpha$ et de la phase mosaïque du système $\mathrm{Sn} / \mathrm{Si}(111)$ résolus angulairement, mesurés le long de la direction [-101] ; b) Spectres de niveaux de coeur Sn 4d correspondants (d'après [15]).
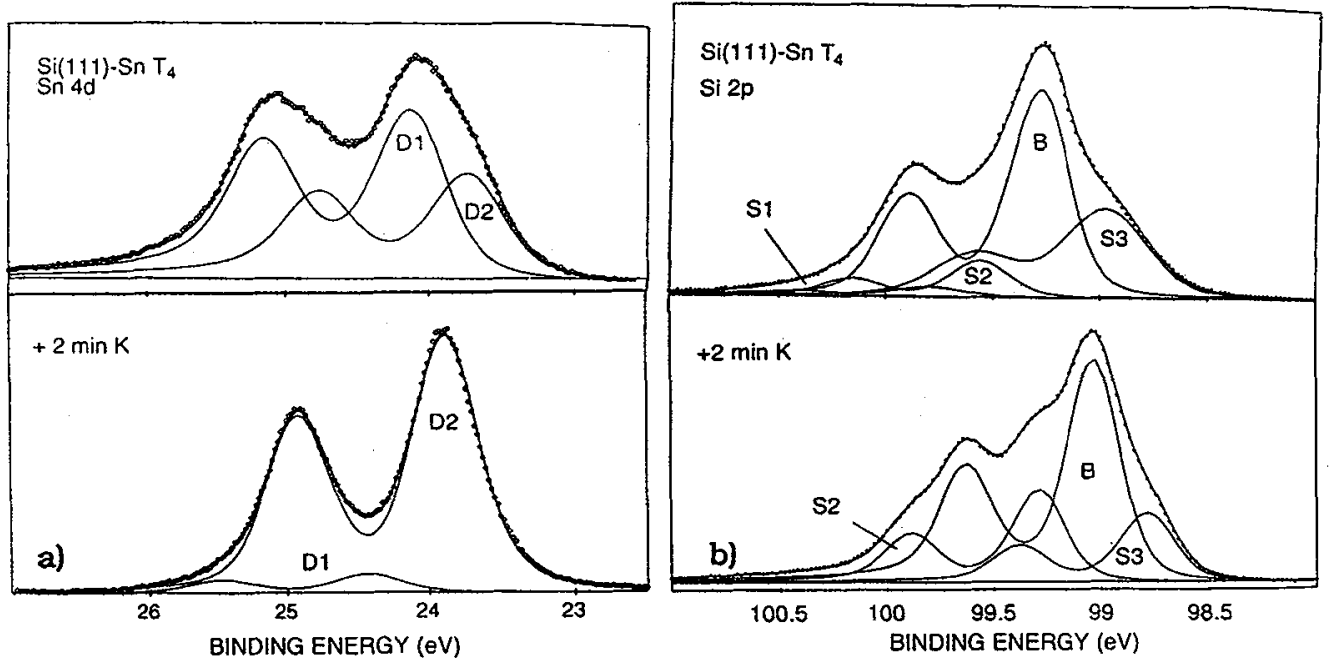

Figure 5 : a) Spectres du niveau de coeur $\mathrm{Sn} 4 \mathrm{~d}$ de la surface $\mathrm{Si}(111) \mathrm{R} 3 \alpha-\mathrm{Sn}$ (haut) puis après adsorption à température ambiante de $\sim 1 / 3 \mathrm{MC}$ de potassium $(\mathrm{hv}=70 \mathrm{eV}) ; \mathrm{b})$ Spectres $S i 2 \mathrm{p}$ correspondants $(\mathrm{hv}=130 \mathrm{eV})$.

Notre interprétation des deux composantes pour la structure $\mathrm{R} 3 \alpha$ renvoie au fait que les adatomes d'étain sont soit dans un état métallique (D1), soit dans un état semiconducteur (D2); dans l'état semiconducteur la liaison pendante de l'atome d'étain est pleine, alors qu'elle est non saturée dans l'état métallique. Comme tous les adatomes apparaissent identiques dans les images de microscopie tunnel, il doit y avoir une oscillation rapide de la charge électronique, par l'intermédiaire des phonons, entre les différents états de liaison pendante. 
La fluctuation entre ces deux états se manifeste également au travers de la largeur très importante du spectre Si 2p pour les atomes de silicium situés sous les adatomes d'étain. Le rôle que jouent les atomes de potassium, qui préservent la structure $\mathrm{R} 3 \alpha$ est celui de donneurs d'électrons remplissant la liaison pendante non saturée, conduisant ainsi la surface métallique $\mathrm{R} 3 \alpha$ vers un état semiconducteur s'apparentant fortement à la phase mosaiqque.

\subsection{Onde de densité de charge de surface à basse température}

La fluctuation de charge mise en évidence pour les systèmes $\mathrm{Sn} / \mathrm{Si}(111)$ et $\mathrm{Sn} / \mathrm{Ge}(111)$ trouve sa contrepartie statique avec le système $\mathrm{Pb} / \mathrm{Ge}(111)$ [2]. Au refroidissement la phase $\mathrm{R} 3 \alpha$, métallique à température ambiante, se transforme progressivement et de façon réversible en une phase semiconductrice $3 \times 3$. La figure 6 illustre cette évolution, montrant le changement de cliché de diffraction d'électrons lents et l'émergence, en spectroscopie de pertes d'énergie, à partir du simple profil décroissant de Drude à température ambiante, d'une structure discrète, à basse température, traduisant la transition de métal à nonmétal. Le spectre mis en insert, enregistré hors de la réflexion spéculaire, montre l'ouverture d'une bande interdite de largeur maximum $65 \mathrm{meV}$.

Tous les atomes de plomb dans les images de microscopie tunnel de la structure R3 $\alpha$ acquises en états pleins ou vides apparaissent identiques.
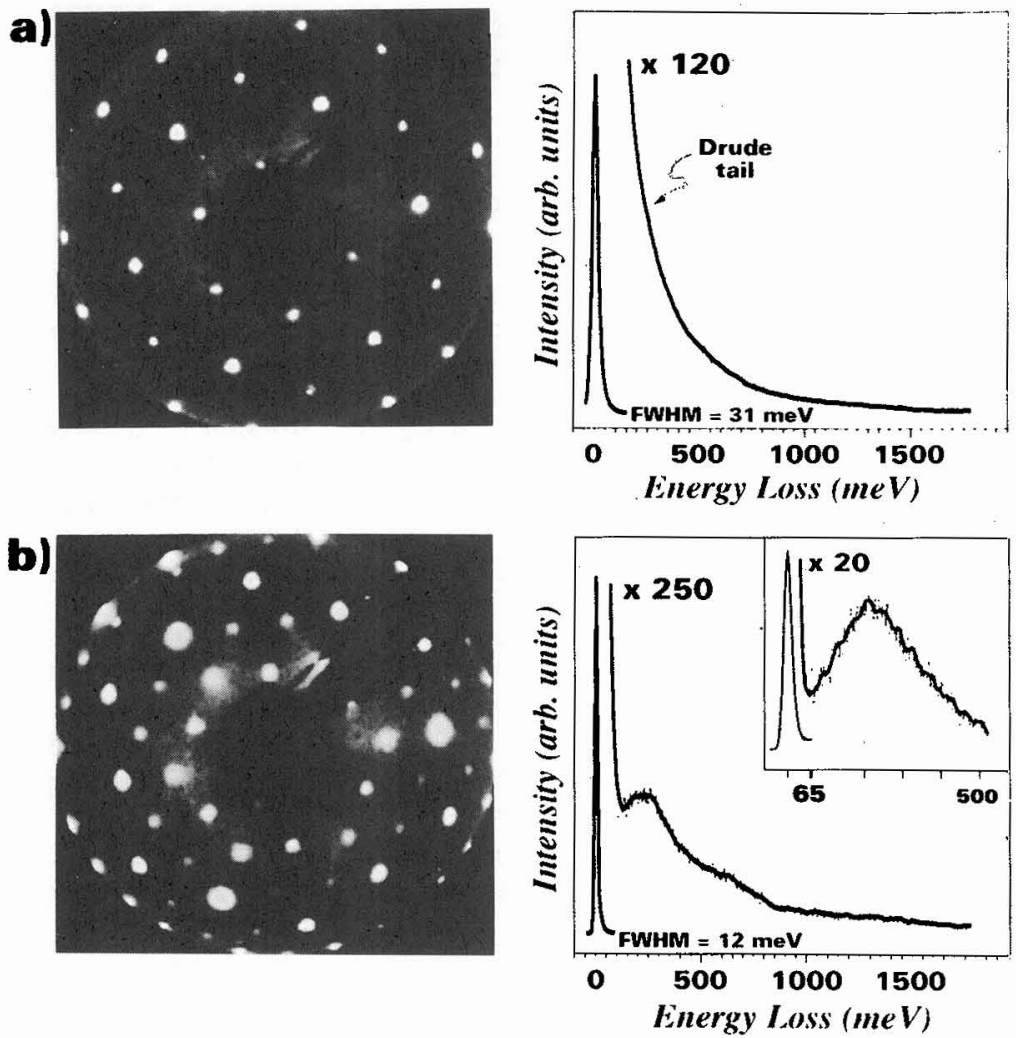

Figure 6 : Clichés de diffraction d'électrons lents $(\mathrm{Ep}=48,6 \mathrm{eV}$ (à gauche) et spectres de pertes d'énergie (à droite) a) de la phase $R 3 \alpha$ à température ambiante et b) de la phase $3 \times 3$ à $\sim 100 \mathrm{~K}$ (d'après [2]). 
Ce n'est plus le cas au contraire pour les images de la structure $3 \times 3$, obtenues à basse température ( $\mathrm{T} \sim 60 \mathrm{~K}$ ) où un réarrangement de la charge sous forme de nid d'abeille est manifeste (fig. 7 ).

a)

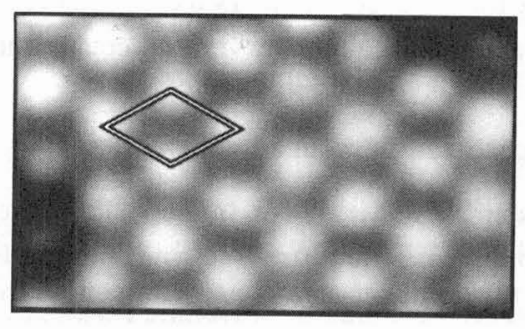

EMPTY STATE

b)

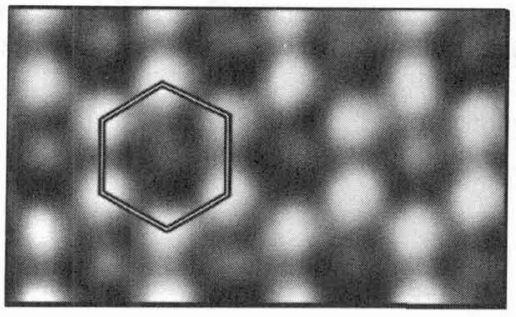

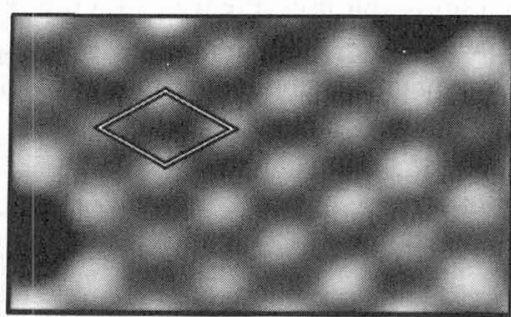

FILLED STATE

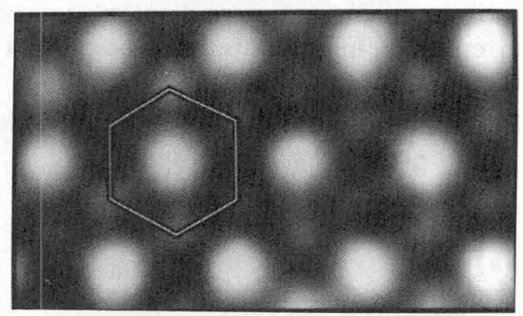

Figure 7 : Images tunnel en états vides (échantillon polarisé à $\mathrm{Vs}=+1 \mathrm{~V}$ ) et états pleins $(\mathrm{Vs}=-1 \mathrm{~V})$ a) de la phase $\mathrm{Ge}(111) \mathrm{R} 3 \alpha-\mathrm{Pb}$ à température ambiante et $\mathrm{b}$ ) après refroidissement à basse température : $\mathrm{T} \sim 60 \mathrm{~K}$ où l'on observe un cliché de diffraction d'électrons lents $3 \times 3$ (d'après [2].

Carpinelli et al. [2] interprètent ce curieux comportement par la création d'une onde de densité de charge de surface commensurable, qui serait la première mise en évidence directe de ce phénomène. En d'autres termes, une redistribution périodique de la charge de valence, abaissant la symétrie, conduit à un réarrangement des bandes électroniques et de ce fait à une diminution de l'énergie totale ; comme d'habitude, cette réorganisation de la charge induit une légère distorsion périodique du réseau. La transition serait analogue à la distortion de Peirls bien connue pour les systèmes unidimensionnels. La "mer" de liaisons pendantes à demi pleine pour la phase R3 $\alpha$ à température ambiante est intrinsèquement instable. Au refroidissement, quand l'agitation thermique est réduite, les électrons se réorganisent par le biais du couplage electron-phonon qui résulte d'une surface de Fermi fortement emboitée. Comme dans le cas uni-dimensionnel, la cellule unité de ce système à basse température correspond à la réorganisation commensurable la plus petite possible ; à une dimension c'est le doublement du paramètre de réseau qui conduit à un nombre pair d'électrons par cellule élémentaire et à un état fondamental non-métallique. Pour le réseau triangulaire à deux dimensions de la phase $\mathrm{R} 3 \alpha$, la maille unité est triple aprés la transition, ce qui conduit à un nombre impair d'électrons par maille élémentaire et donc mènerait normalement à une surface métallique si d'importants effets de corrélations electron-electron n'entraient pas en jeu pour stabiliser la phase $3 \times 3$ consécutivement à la formation de l'onde de densité de charge de surface.

Revenant à l'élargissement surprenant des niveaux de coeur $\mathrm{Pb} 5 \mathrm{~d}$ lors de la formation de la phase $\mathrm{R} 3 \alpha$, comme nous l'avons discuté plus haut, nous pouvons en trouver une explication raisonnable en admettant que cet élargissement provient sans doute de stades préliminaires à la transition de métal à non-métal et d'états précurseurs de la formation de l'onde de densité dencharge de surface. Deux indications vont dans ce sens. La première est l'observation d'une augmentation importante de la largeur de la Gaussienne des raies $\mathrm{Ge} 3 \mathrm{~d}$ du substrat (de $0,31 \mathrm{eV}$ à $0,392 \mathrm{eV}$ selon Carlisle et al. [11]); parallèlement à l'élargissement des raies $\mathrm{Pb} 5 \mathrm{~d}$, si l'on se rappelle notre discussion des systèmes $\mathrm{Si}(111), \mathrm{Ge}(111) \mathrm{R} 3 \alpha-\mathrm{Sn}$, ceci semble indiquer que la phase $\mathrm{Ge}(111) \mathrm{R} 3 \alpha-\mathrm{Pb}$ hésite en quelque sorte entre son état métallique à température ambiante et un état fondamental semiconducteur plus stable. La seconde provient du caractère graduel de la transition qui se manifeste en diffraction d'électrons lents dés $-20^{\circ} \mathrm{C}$ (apparition de tâches d'ordre $1 / 3$ associées à la structure $3 \times 3$ ). Il est donc probable que des états précurseurs au développement de l'onde de densité de charge de surface préexistent déjà à température ambiante. 


\section{STRUCTURES INDUITES PAR Pb ET Bi SUR Si(100) A FAIBLE RECOUVREMENT}

Les premiers stades de l'adsorption de $\mathrm{Pb}$ sur la surface propre $\mathrm{Si}(100)$ reconstruite $2 \times 1$ ont été étudiés récemment par Veuillen et al. [3]. On peut voir sur la figure 8 de longues chaines unidimensionnelles rectilignes de dimères dè plomb orientées perpendiculairement à la direction des rangées sous-jacentes de dimères de silicium.
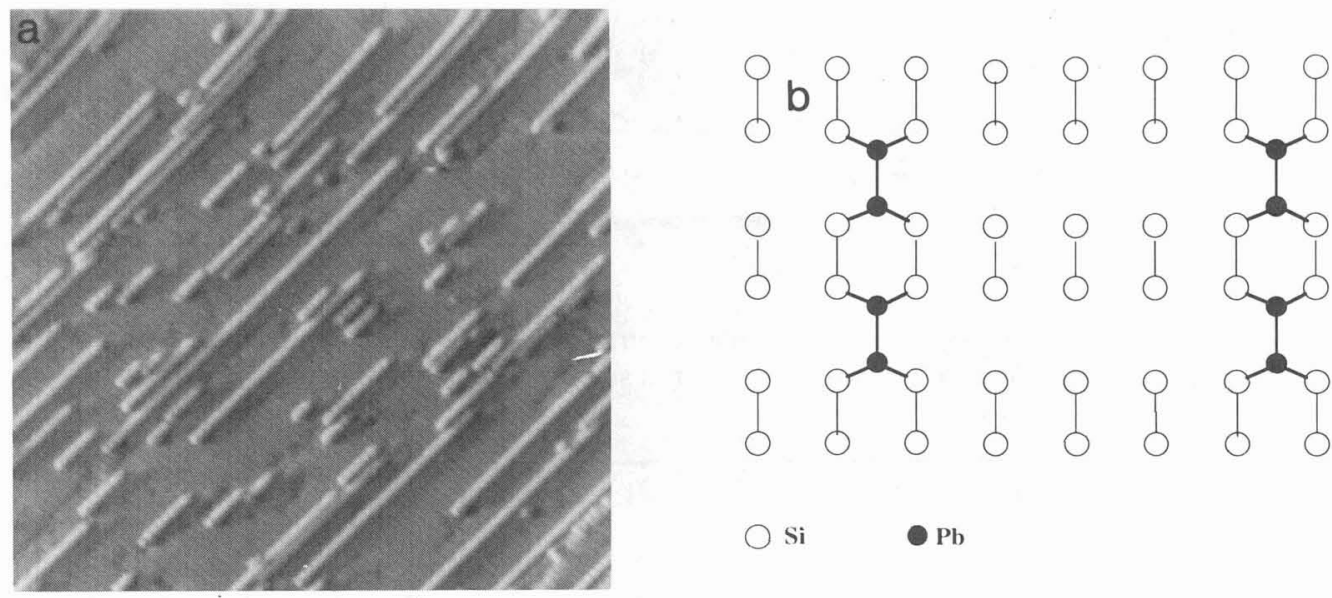

Figure 8 : a) Image de microscopie tunnel en états pleins (polarisation de l'échantillon $-2 \mathrm{~V}$, courant tunnel $0,2 \mathrm{nA}$ ) correspondant à un dépôt de 0,06 MC de plomb sur $\mathrm{Si}(100) 2 \times 1$ à température ambiante (taille de l'image : $46 \times 46 \mathrm{~nm}^{2}$ ) ; b) Modèle géométrique de dimères de plomb parallèles aux dimères de silicium (d'après [3]).

Le mécanisme de formation de ces longues chaines sur $\operatorname{Si}(100) 2 \times 1$ a été étudié théoriquement par Brocks et al. [16] dans le cas de l'aluminium et décrit en termes de "réaction de polymérisation de surface" : quand un dimère d'aluminium isolé se forme (en orientation parallèle comme indiqué sur la figure $8 \mathrm{~b}$ ), les deux atomes d'Al sont liés à quatre atomes de silicium appartenant à quatre dimères de silicium situés sur des rangées adjacentes. La faible liaison $\pi$ du dimère de silicium est alors rompue, ce qui rend quatre liaisons pendantes aux quatre atomes de silicium qui ne sont pas directement liés à l'aluminium. Ces liaisons pendantes sont des sites d'adsorption préférentiels pour le métal, ce qui permet la propagation de la structure en chaine, pour peu que la diffusion du métal soit assez rapide. Il serait bien sûr très intéressant d'observer ces chaines unidimensionnelles à basse température et peut être de mettre en évidence une éventuelle distorsion de Peierls ...

Pour un recouvrement d'environ une monocouche $\mathrm{Li}$ et al. [4] ont obtenu à température ambiante des images de la structure $2 \times 1-\mathrm{Pb}$, pour laquelle apparemment des dimères de plomb formant de longues chaines adjacentes sont bien mis en évidence.

Le mécanisme de formation des chaines invoqué plus haut militerait en faveur de la disparition des dimères de silicium. Nous en avons quelque indication dans les spectres de photoémission des niveaux de coeur Si 2p du substrat (mesurés il y a quelques années avec une assez piètre résolution) [17]. En effet, la composante de surface située du côté des plus faibles énergies de liaisons (énergies cinétiques plus élevées) parait disparaitre sous la couche de dimères de plomb comme le montre le spectre de différence de la figure 9. 


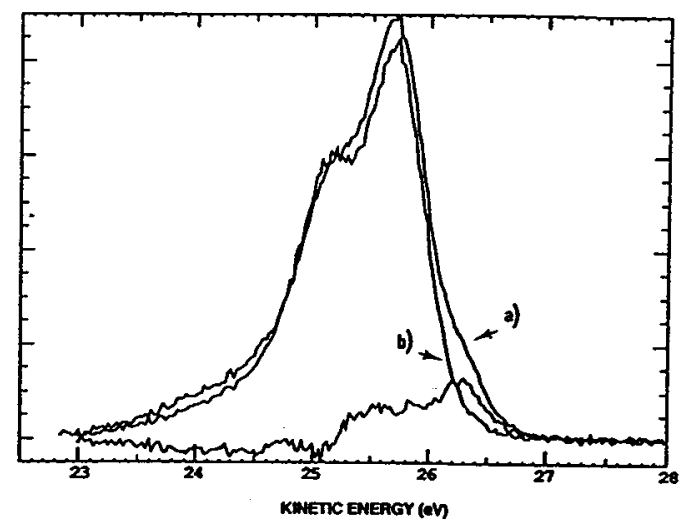

Figure 9 : Spectres du niveau de coeur Si $2 \mathrm{p}$ en mode de surface $(\mathrm{hv}=130 \mathrm{eV})$, à moyenne résolution, a) pour la surface $\mathrm{Si}(100) 2 \times 1$ propre, b) pour la surface recouverte de plomb $\mathrm{Si}(100) 2 \times 1-\mathrm{Pb}$ et spectre de différence $a-b$

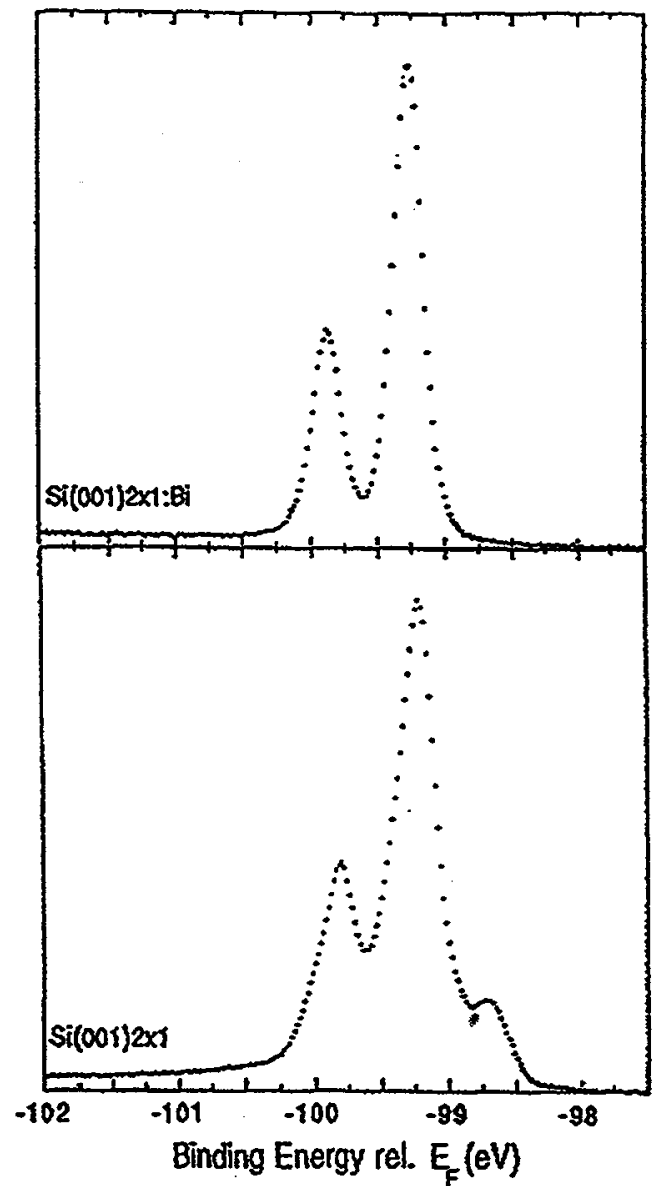

Figure 10 : Spectres du niveau de coeur Si $2 p$ en haute résolution en mode sensible à la surface $(\mathrm{hv}=130 \mathrm{eV})$ pour des substrats de type $n$; $\mathrm{Si}(100) 2 \times 1$ propre en bas, $\mathrm{Si}(100) 2 \times 1-\mathrm{Bi}$ au-dessus. 
Toutefois l'effet est beaucoup plus spectaculaire dans le cas comparable du bismuth, la mesure ayant été effectuée en très haute résolution ( $70 \mathrm{meV})$ à MAX-Lab [5]. La figure 10 confirme que les dimères de $\mathrm{Bi}$ constitutifs de la structure $2 \times 1$ à saturation reposent sur un substrat non reconstruit. En effet le spectre Si 2 p associé ne comporte qu'une seule composante étroite correspondant à la composante de volume du substrat. Ceci est bien en accord avec la faible différence des électronégativités de Si et Bi et corrobore le modèle de Noh et al. [18] déduit de leurs observations en microscopie tunnel (figure 11) en révélant que les atomes de silicium de surface sont complètement relaxés vers des positions de type volume ; les dimères de silicium de la surface vierge initiale sont donc rompus. On notera cependant par ailleurs que contrairement au cas du plomb les dimères de bismuth dans la structure Si(100)2x1-Bi sont orthogonaux, et non parallèles, aux dimères initiaux de silicium de la surface $\mathrm{Si}(100) 2 \times 1$ vierge.

Bien évidemment des calculs comparatifs de minimisation de l'énergie totale seraient souhaitables pour déterminer le pourquoi de cette différence d'orientation.

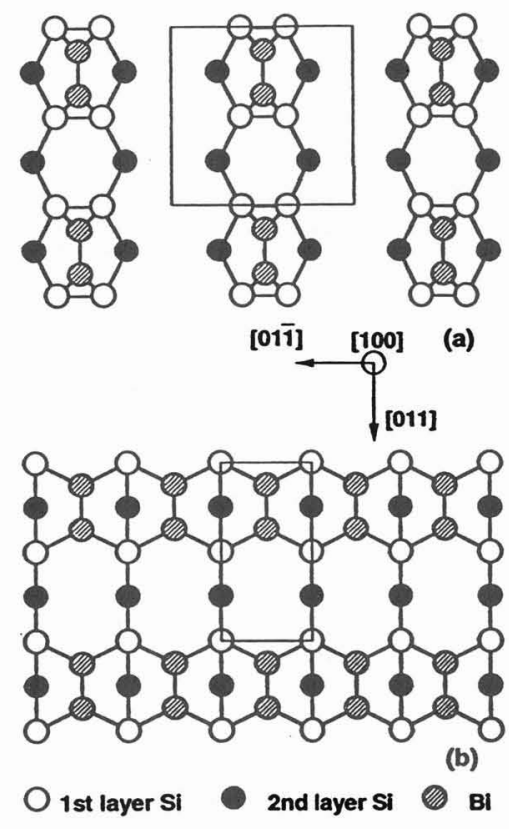

Figure 11 : Modèles atomiques. a) de la structure $\mathrm{Si}(100) 2 \times 1-\mathrm{Bi}$ à faible taux de recouvrement et b) de la structure $2 \times 1$ à saturation. Les rangées de dimères sont respectivement selon les directions [001] pour Si et [01-1] pour $\mathrm{Bi}$ (d'aprés [19]).

\section{STRUCTURE INTERFACIALE ET BARRIÈRE DE SCHOTTKY}

L'identification des mécanismes fondamentaux qui déterminent la hauteur de la barrière de potentiel (barrière de Schottky) aux interfaces métal/semiconducteur reste un vrai défi. Dans un travail de pionnier, Tung [20] a montré que cette hauteur de barrière dépend de la cristallographie interfaciale. Le même effet a été démontré pour des interfaces $\mathrm{Pb} / \mathrm{Si}(111)$ : pour des diodes réalisées à partir d'une surface initiale vierge $\mathrm{Si}(111) 7 \times 7$ les mesures électriques conduisent à une hauteur de barrière (contact redresseur) de $0,7 \mathrm{eV}$ alors que cette même hauteur devient $0,93 \mathrm{eV}$ si la surface initiale est la structure $\mathrm{R} 3 B$ préparée par dépôt d'une couche bidimensionnelle de plomb $(4 / 3 \mathrm{MC})$ et recuit [6]. Comme dans les deux cas l'orientation du film épais de plomb est la même, la différence de hauteur de barrière ne peut provenir que de différentes structures atomiques aux interfaces enterrés. C'est exactement ce que des études de diffraction X corroborent : la reconstruction $7 \times 7$ reste essentiellement inchangée même après recuit à $\sim 150^{\circ} \mathrm{C}[21]$ alors qu'au contraire la structure R3B elle ne perdure pas lors de la condensation d'une couche épaisse à température ambiante [22]. 

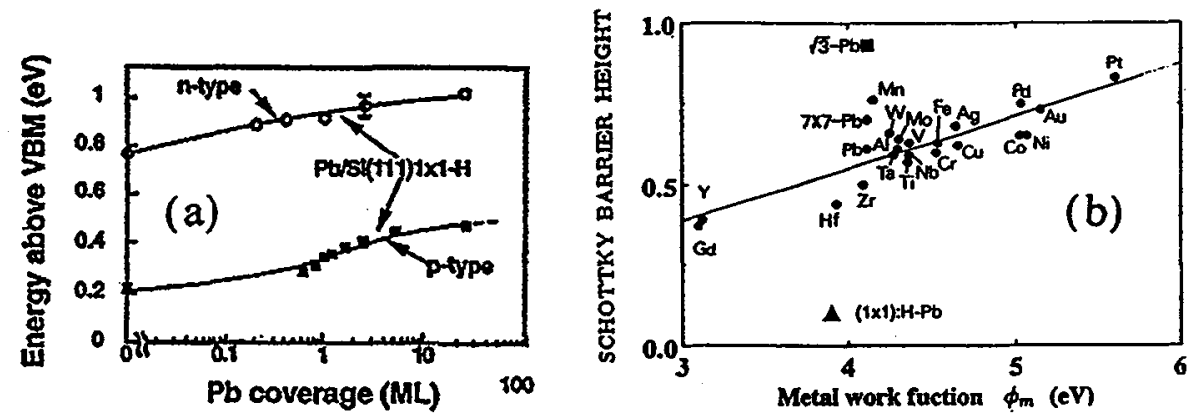

Figure 12 : a) Mouvement du niveau de Fermi, mesuré par photoemission, lors du dépôt de plomb sur la surface Si(111)1 1 1-H (substrats n et p) préparée par voie chimique, les positions initiales correspondent à des bandes plates [7] ; b) Hauteurs de barrière pour des contacts métal/n-Si(111) en fonction du travail de sortie du métal ; les labels $\sqrt{3}-\mathrm{Pb}, 7 \times 7-\mathrm{Pb}$ et $(1 \times 1): \mathrm{H}-\mathrm{Pb}$ indiquent des contacts réalisés à partir de la surface $\mathrm{R} 3 \mathrm{~B}$, de la surface vierge, et de la surface idéalement terminée par l'hydrogène. Les valeurs des hauteurs de barrières sont tirées de [6], [7] et [27], celles des travaux de sortie de [28] hormis pour 1x1:H-Pb où la valeur choisie est celle calculée par Kiejna [29].

La présence d'hydrogène à l'interface a une incidence énorme. Ainsi nous avons montré (fig. 12), en suivant par photoémission du niveau de coeur Si $2 p$, la variation de courbure de bande, qu'en partant de conditions initiales de bandes plates à la surface $\mathrm{Si}(111) 1 \times 1-\mathrm{H}$, idéalement terminée par de l'hydrogène (préparation par voie aqueuse selon la méthode de Higashi et al. [23], nous arrivions lors du dépôt de plomb à amener le niveau de Fermi dans la bande interdite à proximité $(\sim 100 \mathrm{meV})$ du bas de la bande de condution. Un tel comportement devrait induire un contact ohmique pour des diodes $\mathrm{Pb} / \mathrm{n}-\mathrm{Si}(111) 1 \times 1-\mathrm{H}$ à condition que l'hydrogène reste fixé à l'interface lors de la croissance du film épais de plomb. Mönch et al. pensent avoir obtenu de tels contacts ohmiques [24] mais ce résultat est contesté par Osvald et al. [8, 25] qui ont obtenu des contacts redresseurs probablement en raison d'une perte d'hydrogène à l'interface comme c'est effectivement le cas lors de la réalisation de diodes $\mathrm{Ag} / \mathrm{Si}(111) 1 \times 1-\mathrm{H}$ [26].

En fait, en raison du caractère inerte de l'interface $\mathrm{Pb} / \mathrm{Si}(111) 1 \times 1-\mathrm{H}$, l'on doit s'attendre, s'il reste stable, à un contact ohmique. En effet, l'on peut supposer qu'alors la hauteur de barrière sera proche de la limite de Schottky-Mott déterminée par la différence entre le travail de sortie du métal et l'affinité électronique $\chi$ du semiconducteur. Dans notre cas ceci conduirait à :

$\Phi_{\mathrm{b}, \mathrm{n}}=\Phi_{\mathrm{Pb}}-\chi$, soit $\Phi_{\mathrm{b}, \mathrm{n}}=0,07 \mathrm{eV}$,

avec $\Phi_{\mathrm{Pb}}=3,9 \mathrm{eV}$ [29] et $\chi=3,83 \mathrm{eV}$ pour $\mathrm{Si}(111) 1 \times 1-\mathrm{H}$ [30], en excellent accord avec nos résultats de photoémission qui anticipent bien un contact ohmique (sous réserve évidemment d'absence de perte d'hydrogène comme indiqué ci-dessus).

La limite de Schottky-Mott atteinte ainsi pour le silicium est une situation exceptionnelle ; tout autant l'est le fait inédit que pour un seul et unique métal (inerte!), le plomb en l'occurence, la hauteur de barrière de Schottky pour des contacts $\mathrm{Pb} / \mathrm{Si}$ puisse varier de pratiquement zéro (comportement ohmique) à environ 1 $\mathrm{eV}$ selon, en tout et pour tout, la préparation initiale de la surface de départ : terminaison idéale par l'hydrogène ou bien phase $R 3 B$ et donc en fait selon seulement la structure atomique à l'interface : soit $1 \times 1-\mathrm{H}$ (plus ou moins bien préservée) ou simplement $1 \times 1$, puisque la structure $\mathrm{R} 3 B$ disparait sous des films épais.

Un tel comportement est évidemment tout à fait unique : la gamme couverte avec un nombre élevé d'autres métaux sur Si ne couvre qu'environ $45 \%$ de la bande interdite [27] comme on peut le voir sur la figure $12 \mathrm{~b}$.

\section{CONCLUSION}

L'étude de systèmes métal/semiconducteur inertes (dits "prototypes") s'est avérée particulièrement fertile, riche de phénomènes nouveaux, subtils, souvent inattendus. Des développements sont envisageables vers la réalisation d'empilements multicouches qui pourraient peut-être trouver des applications dans le domaine 
de la micro-électronique ou même de la supraconductivité comme peut le laisser entrevoir la mise en évidence de fluctuations métal-semiconducteur et la création d'ondes de densité de charge de surface associées à de fortes corrélations.

\section{Références}

[1] Göthelid M., Björkquist M., Grehk T.M., Le Lay G. et Karlsson U.O., Phys. Rev. B 52 (1995) R 14352

[2] Carpinelli J.M., Weitering H.H., Plummer E.W. et Stumpf R., Nature 381 (1996) 398

[3] Veuillen J.Y., Gomez-Rodriguez J.M. et Cinti R.C., J. Vac. Sci. Technol. B 14 (1996) 1010

[4] Li L., Koziol C., Wurm K., Hong Y., Bauer E. et Tsong T.S.T., Phys. Rev. B 50 (1994) 10834

[5] Håkansson M.C., Johansson L.S.O., Grehk T.M., Göthelid M. Björquist M., Karlsson U.O. et Le Lay G., Phys. Rev. B, sous presse

[6] Heslinga D.R., Weitering H.H., van der Werf D.P., Klapwijk T.M. et Hibma T., Phys. Rev. Lett.. 64 (1990) 1589

[7] Aristov V. Yu, Le Lay G., Hricovini K., Taleb-Ibrahimi A., Dumas P., Günther R., Osvald J. et Indlekofer G., J. Electron Spectrosc. Rel. Phenom. 68 (1994) 419

[8] Osvald J., Hricovini K., Le Lay G. et Aristov V. Yu., Fizika A4 (1995) 191

[9] Le Lay G. dans "Growth and Properties of Ultrathin Epitaxial Layers", The Chemical Physics of Solid Surfaces, vol. 8, D.A. King et D.P. Woodruff eds., Elsevier Science BV, Amsterdam, sous presse

[10] Feidenhans'1 R., Pedersen J.S., Nielsen M., Grey F. et Johnson R., Surf. Sci. 178 (1986) 927

[11] Carlisle J.A., Miller T. et Chiang T.C., Phys. Rev. B 47 (1993) 3790

[12] Carlisle J.A., Miller T. et Chiang T.G., Phys. Rev. B 45 (1992) 3400

[13] Göthelid M., Grehk T.M., Törnevik C., Karlsson U.O. et Flödström S.A., Surf. Sci. 328 (1995) 80

[14] Törnevik C., Göthelid M., Hammar M., Karlsson U.O., Flodström S.A., Wigren C. et Östling M., Surf. Sci. 314 (1994) 179

[15] Karlsson C.J., Thèse de doctorat, Université de Linköping, 1994

[16] Brocks G., Kelly P.J. et Car R., Phys. Rev. Lett. 70 (1993) 2786

[17] Le Lay G., Abraham M., Kahn A., Hricovini K. et Bonnet J.E., Physica Scripta T35 (1991) 261

[18] Noh H.P., Park Ch., Jeon D., Cho K., Hashizume T., Kuk Y. et Sakurai T., J. Vac. Sci. Technol. $B 12$ (1994) 2

[19] Tang S. et Freeman A.J., Phys. Rev. B 50 (1994) 17

[20] Tung R.T., Phys. Rev. Lett. 52 (1984) 461

[21] Lucas C.A. et Loretto D., Surf. Sci. 344 (1995) L 12

[22] Howes P.B., Edwards K.A., Hughes D.J., Mac Donald J.E., Hibma T., Bootsma T. et James M.A., Phys. Rev. B 51 (1995) 17740

[23] Higashi G.S., Chabal Y.J., Trucks G.W. et Raghavachari K., Appl. Phys. Lett. 56 (1990) 651

[24] Mönch W., Phys. Low-Dim. Struct., $4 / 5$ (1994) 1 ; Kampen T.V. et Mönch W., Surf. Sci. $331-$ 333 (1995) 490

[25] Osvald J. et Dobrocka E., Semicond. Sci. Technol. 11 (1996) 1198

[26] Mishiyama A., ter Horst G., Zagwijn P.M., van den Hoven G.N., Frenken J.W.M., Garten F., Schlatmann A.R. et Vrijmoth J., Surf. Sci. 300 ( 1996) 229

[27] Schmid P., Helv. Phys. Acta 58 (1985) 3271

[28] Michaelson H.B., J. Appl. Phys. 48 (1977) 4729 ; IBM J. Res. Der. 22 (1978) 72

[29] Kiejna A., Phys. Rev. B 47 (1993) 7361

[30] Safta N., Lacharme J.P., Sebenne C.A. et Akremi A., J. Phys. Cond. Matter 